\title{
The correlation between blue straggler and binary fractions in the core of Galactic globular clusters ${ }^{\star}$ (Research Note)
}

\author{
A. Sollima ${ }^{1}$, B. Lanzoni ${ }^{1}$, G. Beccari ${ }^{1,2}$, F. R. Ferraro ${ }^{1}$, and F. Fusi Pecci ${ }^{2}$ \\ 1 Dipartimento di Astronomia, Università di Bologna, via Ranzani 1, 40127 Bologna, Italy \\ e-mail: antonio.sollima@oabo.inaf.it (AS) \\ 2 INAF - Osservatorio Astronomico di Bologna, via Ranzani 1, 40127 Bologna, Italy
}

Received 16 November 2007 / Accepted 24 January 2008

ABSTRACT

\begin{abstract}
Context. Blue stragglers stars (BSSs) are thought to form in globular clusters by two main formation channels: $i$ ) mergers induced by stellar collisions; and ii) coalescence or mass transfer between companions in binary systems. The detailed study of the BSS properties is therefore crucial for understanding the binary evolution mechanisms and the complex interplay between dynamics and stellar evolution in dense stellar systems.

Aims. We present the first comparison between the BSS specific frequency and the binary fraction in the core of a sample of Galactic globular clusters, with the aim of investigating the relative efficiency of the two proposed formation mechanisms.

Methods. We derived the frequency of BSSs in the core of thirteen low-density Galactic globular clusters by using deep ACS@HST observations and investigated its correlation with the binary fraction and various other cluster parameters.

Results. We observed a correlation between the BSS specific frequency and the binary fraction. The significance of the correlation increases by including a further dependence on the cluster's central velocity dispersion.

Conclusions. We conclude that the unperturbed evolution of primordial binaries could be the dominant BSS formation process, at least in low-density environments.
\end{abstract}

Key words. techniques: photometric - binaries: general - blue stragglers - stars: population II - globular clusters: general

\section{Introduction}

Blue straggler stars (BSSs) are objects that, in the color-magnitude diagram (CMD) of evolved stellar populations, lie along an extension of the main sequence (MS) in a region that is brighter and bluer than the turn-off (TO). First discovered by Sandage (1953) in M 3, they have been observed in all Galactic globular clusters (GCs; Piotto et al. 2004), in the field population (Carney et al. 2005), and in dwarf galaxies of the local group (Momany et al. 2007).

Their location in the CMD suggests that BSSs have masses of $1.2 \div 1.5 M_{\odot}$, significantly higher than those of normal stars in old stellar systems (like GCs). Thus, they are thought to have increased their mass during their evolution. Two mechanisms have been proposed for their formation: $i$ ) the merger of two stars induced by stellar collision (COL-BSSs; Hills \& Day 1976) and ii) coalescence or mass transfer between two companions in a binary system (MT-BSSs; McCrea 1964). The two formation channels are thought to act with different efficiencies according to the cluster's structural parameters (Fusi Pecci et al. 1992), and they can work simultaneously within the same cluster in different radial regions, corresponding to widely different stellar densities (Ferraro et al. 1997; Mapelli et al. 2006). Indeed, collisions are more frequent in the central region of GCs, because of the high stellar density, while MT-BSSs mainly populate the cluster periphery, where binary systems can evolve more easily in isolation without suffering exchange or ionization due to

\footnotetext{
* Based on ACS observations collected with the Hubble Space Telescope within the observing program GO 10775.
}

gravitational encounters. The whole scenario is further complicated by the cluster dynamical evolution that leads massive systems (like binaries and BSSs) to sink toward the cluster center in a time-scale comparable to the cluster relaxation time.

A possible tool for distinguishing COL-BSSs from MT-BSSs is based on high-resolution spectroscopic analysis. In fact, anomalous chemical abundances are expected at the surface of BSSs resulting from mass-transfer activity (Sarna \& de Greve 1996), while they are not predicted for COL-BSSs (Lombardi et al. 1995). However, such studies have only just become feasible and are limited to only a small number of BSSs in just one cluster (47 Tucanae; Ferraro et al. 2006).

As an alternative way of getting insight into the relative efficiency of the two formation mechanisms, we investigate possible correlations between the BSS population and the host cluster properties.

\section{Observations and data reduction}

The photometric database considered here is the same as the one used in Sollima et al. (2007, hereafter S07) for measuring the frequency of binary systems in the core of thirteen low-density Galactic GCs. It consists of a set of high-resolution images, obtained with the ACS onboard HST, through the F606W $\left(V_{606}\right)$ and $F 814 W\left(I_{814}\right)$ filters. As described in S07, the target clusters are characterized by high Galactic latitude $\left(b>15^{\circ}\right)$, low reddening $(E(B-V)<0.1)$, and low projected central density $\left(\log \rho_{0}^{\prime}<5\right.$, where $\rho_{0}^{\prime}$ is in units of $\left.M_{\odot} \operatorname{arcmin}^{-2}\right)$. These selection criteria were chosen to limit the effects of field 
contamination, differential reddening, and crowding in the determination of the binary fractions. The selected target clusters and their main physical parameters are listed in Table 1 . The central luminosity density $\rho_{0}$ and the absolute $V$ band magnitude $M_{V}$ are from Djorgovski (1993), the age $t_{9}$ is from Salaris \& Weiss $(2002)^{1}$, and the projected central velocity dispersion $\sigma_{\mathrm{v}}$ is from McLaughlin \& Van der Marel (2005). A detailed description of the data reduction and calibration procedure can be found in $\mathrm{S} 07$, together with the resulting CMDs for all the target clusters.

As discussed in S07, the binary sequence is well-defined and distinguishable from the MS, and the fraction of binary systems $\left(\xi_{\text {bin }}\right)$ found within the cluster core radius ranges from about 10 to $50 \%$ (the value of $\xi_{\text {bin }}$ are listed in Table 1). Moreover, a number of BSSs populating the bright part of the CMD is also evident in the data sample. In the following section we describe the adopted procedure for deriving the BSS frequencies in the core of the target clusters.

\section{BSS population selection}

Our primary criterion for defining of the BSS sample is based on the location of stars in the $\left(I_{814}, V_{606}-I_{814}\right)$ CMD. Since these GCs have different distances, reddening, ages, and metallicities, in order to define a homogeneous criterion for the BSS selection, we shifted the CMD of each cluster in magnitude and color to match that of M 55 (see also Ferraro et al. 1995). For minimizing the contamination from sub-giant branch, horizontal branch (HB), and field stars, we limited the analysis to colors bluer than $\left(V_{606}-I_{814}\right)<0.47$ and to magnitudes in the range $15.1<I_{814}<18.1$. The adopted BSS selection box (the same for all clusters) is shown in Fig. 1 and the number of selected BSSs $\left(N_{\mathrm{BSS}}\right)$ in each target cluster is reported in Table 1. To compare the frequency of BSSs in different GCs, the size of the total cluster population must be taken into consideration. Commonly, the number of BSSs is normalized to the number of stars belonging to a given evolutive sequence (usually the HB, or the red giant branch, RGB; Ferraro et al. 1995; Piotto et al. 2004; Leigh et al. 2007). Unfortunately, in some clusters of our sample, the number of HB and RGB stars is very small, which would make the derived BSS frequency highly uncertain. We therefore normalized the number of selected BSSs to the number of MS stars $\left(N_{\mathrm{MS}}\right)$ in the magnitude range $18.1<I_{814}<19.1$ and with colors comprised within $\Delta\left(V_{606}-I_{814}\right)<0.05$ from the cluster MS mean ridge line. Stars in this magnitude range are bright enough to have the same completeness level as BSSs $(\phi>95 \%)$ and to cover a similar mass range in all clusters.

Following the theoretical stellar isochrones by Cariulo et al. (2004) and taking the differences in age and metallicities among the selected GCs into account, the TO mass varies between $0.8 M_{\odot}<M<0.9 M_{\odot}$. Hence, we considered $N_{\text {MS }}$ a good indicator of the size of the sampled cluster population.

The possible effect of background and foreground field star contamination on the BSS and MS selections has been estimated by using the Galaxy model of Robin et al. (2003). For this purpose a synthetic catalog covering an area of 0.5 square degrees centered on each cluster (with the centers taken from Djorgovski \& Meylan 1993) was retrieved, both in the $V$ and the I Johnson-Cousin bands. A subsample of stars scaled to the size of the ACS field of view $\left(202^{\prime \prime} \times 202^{\prime \prime}\right)$ was randomly extracted, and the Johnson-Cousin magnitudes were converted into

\footnotetext{
${ }^{1}$ Since NGC 6981 is not included in the list of Salaris \& Weiss (2002), we converted the ages measured by De Angeli et al. (2005) into the Salaris \& Weiss (2002) scale.
}

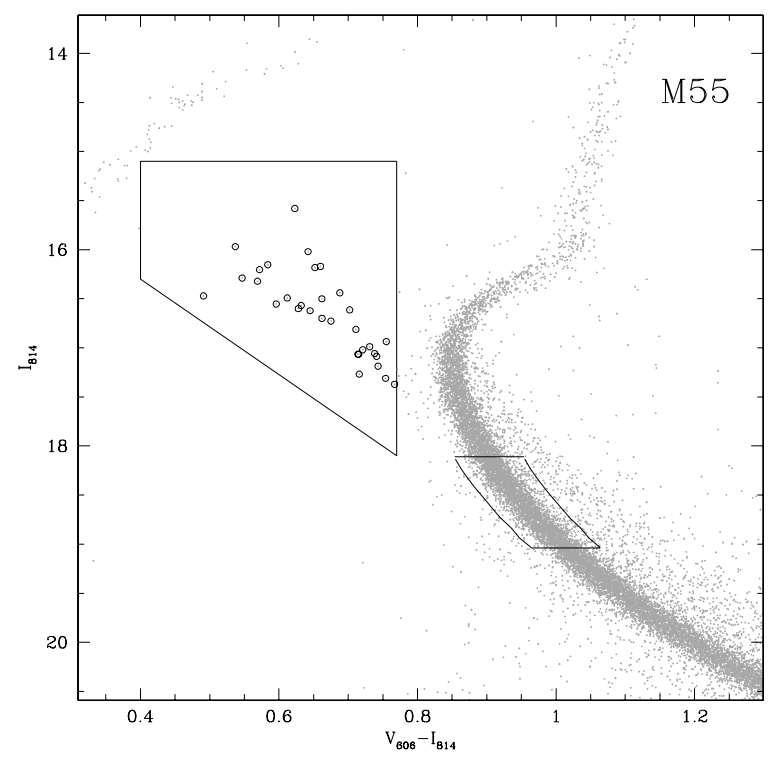

Fig. 1. $\left(I_{814}, V_{606}-I_{814}\right) \mathrm{CMD}$ of M 55. The adopted selection boxes for the BSS and MS populations are shown. The fiducial BSSs are marked with open circles.

Table 1. Main physical parameters of the target globular clusters.

\begin{tabular}{lccccccc}
\hline \hline Name & $\begin{array}{c}\log \rho_{0} \\
L_{\odot} \mathrm{pc}^{-3}\end{array}$ & $M_{V}$ & $\begin{array}{c}t_{9} \\
\mathrm{Gyr}\end{array}$ & $\begin{array}{c}\sigma_{\mathrm{v}} \\
\mathrm{Km} \mathrm{s}^{-1}\end{array}$ & $\begin{array}{c}\xi_{\text {bin }} \\
\%\end{array}$ & $N_{\mathrm{BSS}}$ & $\begin{array}{c}F \\
\%\end{array}$ \\
\hline NGC 288 & 1.80 & -6.63 & 11.3 & 2.79 & 11.6 & 43 & 1.9 \\
NGC 4590 & 2.52 & -7.73 & 11.2 & 3.59 & 14.2 & 62 & 1.5 \\
NGC 5053 & 0.51 & -7.07 & 10.8 & 1.79 & 11.0 & 15 & 1.0 \\
NGC 5466 & 0.68 & -6.83 & 12.2 & 1.86 & 9.5 & 39 & 1.7 \\
NGC 5897 & 1.32 & -7.27 & 12.3 & 2.94 & 13.2 & 53 & 1.3 \\
NGC 6101 & 1.57 & -6.82 & 10.7 & 2.91 & 15.6 & 60 & 1.1 \\
NGC 6362 & 2.23 & -6.69 & 11.0 & 3.83 & 11.8 & 42 & 1.5 \\
NGC 6723 & 2.71 & -7.95 & 11.6 & 5.52 & 16.1 & 35 & 0.5 \\
NGC 6981 & 2.26 & -7.16 & 9.5 & 3.66 & 28.1 & 65 & 1.5 \\
M55 & 2.12 & -7.47 & 12.3 & 3.73 & 9.6 & 32 & 0.8 \\
Arp 2 & -0.35 & -5.30 & 9.7 & 0.72 & 32.9 & 33 & 2.3 \\
Terzan 7 & 1.97 & -5.99 & 7.4 & 2.55 & 50.9 & 33 & 3.4 \\
Palomar 12 & 0.68 & -4.78 & 6.4 & 0.76 & 40.8 & 9 & 2.5 \\
\hline
\end{tabular}

the ACS photometric system by using the transformations of Sirianni et al. (2005). The number of contaminating field stars turns out to be neglegible $(<2)$ in all the clusters of our sample. Then, we counted the number of field stars included in the BSS and MS selection boxes ( $N_{\text {field }}^{\mathrm{BSS}}$ and $N_{\text {field }}^{\mathrm{MS}}$, respectively) and computed the BSS specific frequency as

$F=\frac{N_{\mathrm{BSS}}-N_{\text {field }}^{\mathrm{BSS}}}{N_{\mathrm{MS}}-N_{\text {field }}^{\mathrm{MS}}}$.

The resulting values for the thirteen target clusters are listed in Table 1.

To check the suitability of the adopted normalization criterion, we compared the derived BSS specific frequencies with those measured by Leigh et al. (2007; and updated by Sills 2008, private communication) for the four clusters in common with our sample (namely, NGC 4590, NGC 6362, NGC 6723, and NGC 6981). These authors normalized the number of BSSs in the cluster cores to that of RGB stars in the same magnitude range. In spite of the different normalization criterion, the specific frequencies calculated for the four clusters in common correlate very well (with a correlation coefficient $r=0.92$ ). 
Table 2. Pearson's correlation coefficients $(r)$ and confidence levels $(P)$.

\begin{tabular}{lcc}
\hline \hline Parameter & $r$ & $P$ \\
\hline$\xi_{\text {bin }}$ & 0.820 & 0.9998 \\
$M_{V}$ & 0.774 & 0.9985 \\
$t_{9}$ & -0.758 & 0.998 \\
$\sigma_{\mathrm{v}}$ & -0.594 & 0.98 \\
{$[\mathrm{M} / \mathrm{H}]$} & 0.403 & 0.92 \\
$\rho_{0}$ & -0.311 & 0.85 \\
$r_{\mathrm{h}}$ & 0.299 & 0.84 \\
$\Gamma$ & -0.158 & $<0.75$ \\
$c$ & 0.129 & $<0.75$ \\
$\nu$ & 0.099 & $<0.75$ \\
$\log t_{\mathrm{rh}}$ & 0.020 & $<0.75$ \\
\hline
\end{tabular}

\section{Correlations between BSS specific frequency and cluster parameters}

With the aim of shedding light on the BSS formation mechanisms, we correlated the BSS specific frequency $(F)$ calculated above with the core binary fraction $\left(\xi_{\text {bin }}\right)$ obtained by S07 and with various other physical parameters of the thirteen GCs of the sample. In particular, we searched for possible correlations with the cluster central density, total magnitude, age, and central velocity dispersion (see Table 1), as well as with the stellar collision rate (defined as $\Gamma \equiv \rho_{0}^{2} r_{\mathrm{c}}^{3} / \sigma_{\mathrm{v}}$; Pooley \& Hut 2006), the global metallicity ([M/H], from Ferraro et al. $\left.1999^{2}\right)$, the cluster evaporation rate ( $v$, from Gnedin \& Ostriker 1997), concentration ( $c$, from Trager et al. 1995), half-mass relaxation time $\left(t_{\mathrm{rh}}\right)$, and half-mass radius ( $r_{\mathrm{h}}$, from Djorgovski 1993).

The Pearson's linear correlation coefficients and confidence levels for the considered parameter pairs are listed in Table 2. The strongest correlation is found between the BSS specific frequency and the binary fraction. A noticeable correlation with the absolute magnitude and anticorrelations with the cluster age and central velocity dispersion are also apparent. Considering that the age estimates are quite uncertain and span a narrow range of values in our sample, any firm conclusion about the possible dependence of $F$ on the cluster age is premature at the moment. However, if confirmed, such an anticorrelation might be a consequence of the analogous anticorrelation between binary fraction and age already discussed by S07, and would suggest that binary disruption processes in the core of GCs became more efficient with time, thus reducing the fraction of both binaries and BSS in the core of the oldest clusters (as suggested by Ivanova et al. 2005, but at odds with Hurley et al. 2007). In any case, even if the anticorrelation between $F$ (or $\xi_{\text {bin }}$ ) and $t_{9}$ is not confirmed, the correlation between the BSS specific frequency and the binary fraction still holds. The latter is shown in the upper panel of Fig. 2 and it suggests that the main formation channel of BSS in these clusters is the unperturbed evolution of primordial binary systems.

We stress that, since the considered parameters do not constitute an orthonormal basis, most of the less significant correlations could be artificially induced by primary correlations among other parameters.

To investigate the possible dependence of $F$ on more than one cluster parameter, we applied the Bayesian information criterion (BIC) test (Schwarz 1978) to our dataset. We assumed the

${ }^{2}$ For NGC 6101, NGC 6362, NGC 6723, and Palomar 12 not included in the list of Ferraro et al. (1999), we transformed the metallicity [Fe/H] from Zinn \& West (1984) into the global metallicity [M/H] following the prescriptions of Ferraro et al. (1999).
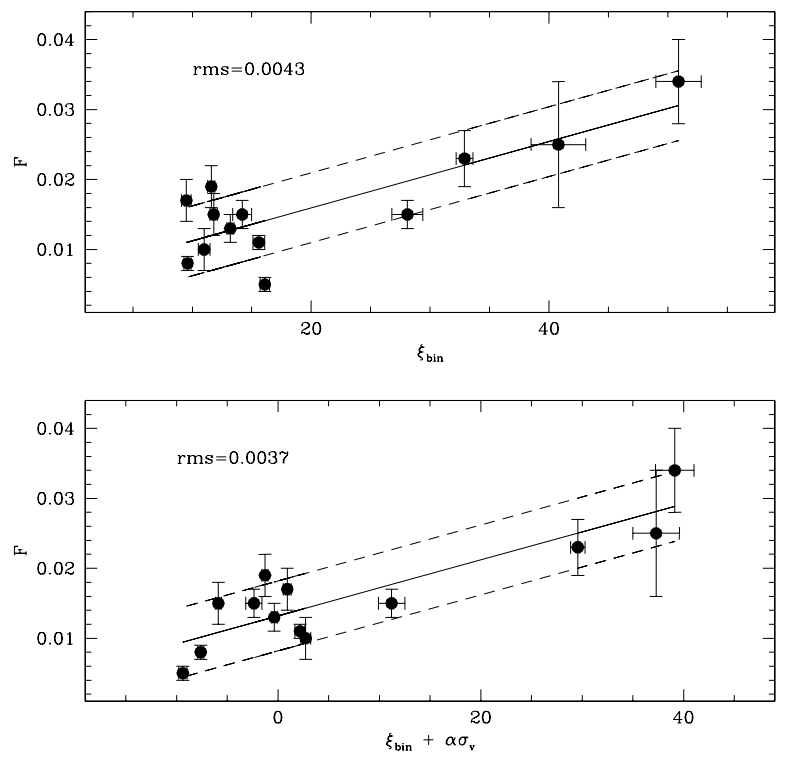

Fig. 2. Specific frequency of BSSs as a function of the core binary fraction (upper panel) and of the best-fit linear combination of core binary fraction and cluster velocity dispersion (bottom panel, obtained with $\alpha=-4.62$ ). Solid lines indicate the best-fit lines; dashed lines mark the boundaries of the \pm 0.005 range in $F$ with respect to the best-fit lines.

BSS specific frequency $F$ to be a linear combination of a subsample of $p$ parameters $\left(\lambda_{i}\right)$ selected among those listed above,

$F_{p}^{\mathrm{fit}}=\sum_{i=1}^{p} \alpha_{i} \lambda_{i}+\alpha_{p+1}$

and we calculated the quantity

$B I C=\log L_{p}-\frac{p}{2} \log N$

where $L_{p}$ is the likelihood and $N$ the dimension of the sample ( $N=13$ in our case). The likelihood is calculated as $\log L_{p}=$ $\sum_{j=1}^{N} \log P_{p, j}$, where

$P_{p, j}=\frac{\mathrm{e}^{\frac{-\left(F_{j}-F_{p, j}^{\mathrm{fit}}\right)^{2}}{2 \sigma_{F}^{2}}}}{\sigma_{F} \sqrt{2 \pi}}$

with $F_{j}$ the BSS fraction of cluster $j$, and $\sigma_{F}$ the residual of the fit. The $p$ parameters that maximize the quantity $B I C$ are the most probable correlators with $F$. For our dataset, the maximum value of $B I C$ is obtained with $p=2, \lambda_{1}=\xi_{\text {bin }}$, and $\lambda_{2}=\sigma_{\mathrm{v}}$. The correlation between $F, \xi_{\text {bin }}$ and $\sigma_{\mathrm{v}}$ is shown in Fig. 2, together with the best-fit line. All the other 3-variate or higher-order correlations turn out to be insignificant.

As a note of caution, however, it is worth adding that these correlations are essentially due to the four clusters (namely Terzan 7, Palomar 12, Arp 2, and NGC 6981) with the largest $(>20 \%)$ binary fraction, while the other GCs in our sample define a group with an average binary content $\xi_{\text {bin }} \sim 10 \%$ and a BSS frequency $F \sim 1.2 \%$. In particular, all the correlations that we have found are driven mainly by Terzan 7, Palomar 12, and Arp 2, which are the GCs not only with the largest binary and BSS fractions, but also with the lowest absolute magnitudes and the lowest ages. These three clusters are also the farthest from the Sun and they are thought to belong to the Sagittarius Stream (Bellazzini et al. 2003). Thus, they might be stellar systems with intrinsically different origins and properties, and the 
correlations found here might not be appropriate for the overall class of "genuine" Galactic GCs. Even when excluding these three clusters from the sample, the anticorrelation with the total luminosity still holds (even if with lower significance), in agreement with what has already been found by Piotto et al. (2004), Sandquist (2005), and Leigh et al. (2007) for larger samples of GCs with different characteristics and for BSS populations selected and normalized with different criteria. Instead, the anticorrelation with the age disappears, thus bringing our results in alignment with those of Leigh et al. (2007; see also De Marchi et al. 2006). The correlation between the BSS and the binary fractions also disappears, excluding the three "Sagittarius" clusters (SgrGCs), with the range in $\xi_{\text {bin }}$ spanned by the remaining sample being very small. Note, however, that both the average BSS and binary frequencies are higher in SgrGCs $\left(\left\langle F_{\mathrm{BSS}}\right\rangle=\right.$ $2.7 \%$ and $\left.\left\langle\xi_{\text {bin }}\right\rangle=41.5 \%\right)$ than in GGCs $\left(\left\langle F_{\mathrm{BSS}}\right\rangle=1.3 \%\right.$ and $\left.\left\langle\xi_{\text {bin }}\right\rangle=14 \%\right)$. Thus, even assuming that SgrGCs were born with different initial conditions from GGCs, the observational facts presented here allow us to draw (at least) the "conservative" conclusion that binary-rich enviroments tend to produce more BSS in the low-density cluster regime (where collisions are expected to play a minor role in the formation/destruction of binaries).

\section{Conclusions}

We measured the BSS specific frequency in the core of thirteen low-density Galactic GCs and investigated its correlation with different dynamical and general cluster parameters. We found evidence that, at least in this density regime, binary-rich environments are more efficient in producing BSS. No correlations have been found with the cluster central density, concentration, stellar collision rate, and half-mass relaxation time, in agreement with the results of Piotto et al. (2004) and Leigh et al. (2007). This evidence indicates that the collisional channel for the BSS formation has a very low efficiency in low-density GCs, while the mechanisms involving the unperturbed evolution of binary systems are dominant.

The higher significance of the trivariate correlation among the BSS frequency, the binary fractions, and the cluster velocity dispersion indicates that, for a given binary fraction, the BSS specific frequency decreases with increasing velocity dispersion. This finding might be connected with the effect of the cluster velocity dispersion in the dynamical evolution of binary systems. In fact, a small cluster velocity dispersion corresponds to a lower energy limit between soft and hard binaries ${ }^{3}$, i.e., to a larger fraction of hard binary systems. Since the natural evolution of hard binaries is to increase their binding energy (i.e. decrease their orbital separation; Heggie 1975), this implies that low-velocity dispersion GCs should host a larger fraction of hard (and close) binaries, able to both survive possible stellar encounters and to activate mass-transfer and/or merging processes between the companions. A larger fraction of BSSs formed by the evolution of primordial binaries is therefore expected in lower velocity dispersion GCs (see also Davies et al. 2004). Such an effect of $\sigma_{\mathrm{v}}$ (in terms of both hardening and shrinking the binary systems) might, in turn, be at the origin of the inverse correlation between the BSS frequency and the cluster total luminosity (mass) observed in open clusters (De Marchi et al. 2006), low-density GCs (Sandquist 2005), as well as high-density GCs

\footnotetext{
${ }^{3}$ A binary is defined soft (hard) if its binding energy $(E=$ $-G m_{1} m_{2} / 2 a$, with $a$ being the orbital separation of the two components) is smaller (larger) than the mean kinetic energy of normal cluster stars ( $K=m \sigma_{\mathrm{v}}^{2}$, with $m$ being the average mass of cluster stars).
}

(Piotto et al. 2004; Leigh et al. 2007). Indeed, the more massive GCs have larger central velocity dispersions (as a consequence of the virial theorem for systems with similar radii, as GCs; see Fig. 1 of Djorgovski 1995). For most of these clusters, however, the binary fraction is still unknown, and the trivariate correlation between $F, \xi_{\text {bin }}$ and $\sigma_{\mathrm{v}}$ cannot be derived. If its significance and its interpretation in terms of the velocity dispersion effect is also confirmed in high-density clusters, then stellar collisions might play a secondary role in the production of BSSs, and the evolution of primordial binaries should always be the dominant process. For the moment, however, these conclusions remain speculative, since the sample of GCs analyzed here, in spite of being the largest to date with known binary fraction, is still too small for statistically reliable assessments. Enlarging the sample of GCs with known binary and BSS fractions is therefore essential and urgent for verifying the findings presented in this paper on a more robust statistical basis.

Acknowledgements. This research was supported by the Ministero dell'Istruzione, Università e Ricerca, and the Agenzia Spaziale Italiana. This research is part of the Progetti strategici di Ateneo 2006 granted by the Bologna University. We warmly thank Paolo Montegriffo for assistance during catalogs cross-correlation. We also thank Alison Sills, the referee of our paper, for her helpful comments and suggestions.

\section{References}

Bellazzini, M., Ferraro, F. R., \& Ibata, R. 2003, AJ, 125, 188 Cariulo, P., Degl'Innocenti, S., \& Castellani, V. 2004, A\&A, 421, 1121

Carney, B. W., Latham, D. W., \& Laird, J. B. 2005, AJ, 129, 466 Davies, M. B., Piotto, G., \& De Angeli, F. 2004, MNRAS, 348, 129 De Angeli, F., Piotto, G., Cassisi, S., et al. 2005, AJ, 130, 116

De Marchi, F., de Angeli, F., Piotto, G., Carraro, G., \& Davies, M. B. 2006, A\&A, 459, 489

Djorgovski, S. 1993, in Structure and Dynamics of Globular Clusters, ed. S. Djorgovski, \& G. Meylan, ASP Conf. Ser., 50, 373

Djorgovski, S. 1995, ApJ, 438, L29

Djorgovski, S., \& Meylan, G. 1993, in Structure and Dynamics of Globular Clusters, ed. S. Djorgovski, \& G. Meylan, ASP Conf. Ser., 50, 325

Ferraro, F. R., Fusi Pecci, F., \& Bellazzini, M. 1995, A\&A, 294, 80

Ferraro, F. R., Paltrinieri, B., Fusi Pecci, F., et al. 1997, A\&A, 324, 915

Ferraro, F. R., Messineo, M., Fusi Pecci, F., et al. 1999, AJ, 118, 1738

Ferraro, F. R., Sabbi, E., Gratton, R., et al. 2006, ApJ, 647, L53

Fusi Pecci, F., Ferraro, F. R., Corsi, C. E., Cacciari, C., \& Buonanno, R. 1992, AJ, 104, 1831

Gnedin, O. Y., \& Ostriker, J. P. 1997, ApJ, 474, 223

Heggie, D. C. 1975, MNRAS, 173, 729

Hills, J. G., \& Day, C. A. 1976, ApL, 17, 87

Hurley, J. R., Aarseth, S. J., \& Shara, M. M. 2007, ApJ, 665, 707

Ivanova, N., Belczynski, K., Fregeau, J. M., \& Rasio, F. A. 2005, MNRAS, 358, 572

Leigh, N., Sills, A., \& Knigge, C. 2007, ApJ, 661, 210

Lombardi, J. C., Jr., Rasio, F. A., \& Shapiro, S. L. 1995, ApJ, 445, L117

Mapelli, M., Sigurdsson, S., Ferraro, F. R., et al. 2006, MNRAS, 373, 361

McCrea, W. H. 1964, MNRAS, 128, 147

McLaughlin, D. E., \& Van der Marel, R. P. 2005, ApJS, 161, 304

Momany, Y., Held, E. V., Saviane, I., et al. 2007, A\&A, 468, 973

Piotto, G., De Angeli, F., King, I. R., et al. 2004, ApJ, 604, L109

Pooley, D., \& Hut, P. 2006, ApJ, 646, L143

Robin, A. C., Reilé, C., Derrière, S., \& Picaud, S. 2003, A\&A, 409, 523

Salaris, M., \& Weiss, A. 2002, A\&A, 388, 492

Sandage, A. R. 1953, AJ, 58, 61

Sandquist, E. L. 2005, ApJ, 635, L73

Sarna, M. J., \& de Greve, J.-P. 1996, QJRAS, 37, 11

Schwarz, G. 1978, Ann. Stat., 6, 461

Sirianni, M., Jee, M. J., Benítez, N., et al. 2005, PASP, 117, 1049

Sollima, A., Beccari, G., Ferraro, F. R., Fusi Pecci, F., \& Sarajedini, A. 2007, MNRAS, 380, 781

Trager, S. C., King, I. R., \& Djorgovski, S. 1995, AJ, 109, 218

Zinn, R., \& West, M. J. 1984, ApJS, 55, 45 